\title{
Structural Principles of Protein Kinase Regulation
}

Frank Sicheri Lunenfeld-Tanenbaum Research Institute, Mount Sinai Hospital

Protein kinases, through their ability to alternate between catalytically active and inactive states, act as the molecular switches that regulate virtually every cellular process.

Detailed studies of a subset of all protein kinases have uncovered diverse mechanisms of action and regulation despite a high degree of structural conservation within their catalytic domains. In the Sicheri lab, research efforts are focused on the structural and functional characterization of novel protein kinase regulatory mechanisms and mechanisms of dysregulation that give rise to disease. Recent advances in our progress will be presented. 